\title{
COVID-19 in Kerala: Health Index Theory
}

\author{
Zameer Shervani
}

\section{ABSTRACT}

The recent surge in COVID-19 cases in the state of Kerala in India has been studied in this article. The dynamics of virus spread, sero-survey results, health index, and herd immunity concept have been applied. Kerala, with the highest score on the health index among all the states of India, is most susceptible to the SARS-CoV-2 virus. Kerala has nearly the same number of cases as Germany. The "Health Index Theory" established in this review article first has explained well the surge in the cases. Briefly, the concept of COVID-19 herd immunity has also been tested first.

Keywords: Kerala COVID-19, Sero-survey, Health index theory, Herd immunity.

Submitted : March 24, 2021

Published : April 15, 2021

ISSN: 2593-8339

DOI: $10.24018 /$ ejmed.2021.3.2.781

Zameer Shervani*

Food \& Energy Security Research \& Product Centre, Sendai, Japan. (e-mail: shervani.nanotek@gmail.com)

*Corresponding Author

\section{INTRODUCTION}

The origin of the COVID-19 pandemic, infection aspect, and testing and treatment have been described in recently published articles [1]-[3]. In article [3], the initial attempts and success of COVID-19 vaccines have been compared. Japanese researchers [4] employed the world's fastest supercomputer "Fugaku" to pick suitable drugs from the existing 2128 drugs' stock for COVID-19 treatment. Using the "Fugaku" supercomputer, "3 Cs" protocols (avoid closed spaces, crowded places, and close contacts) were established to limit the pandemic. Inhaling a large viral load of airborne COVID-19 virus is avoided if $3 \mathrm{Cs}$ are observed. The SARS-CoV-2 reverse-zoonotic transmission from humans to household pets has been established [5]. Though, the zoonotic transmission from household pets to humans was not noticed except in mink. However, it was suggested to isolate and quarantine pets similar to humans when they tested positive or developed symptoms. Cats and ferrets were susceptible to the SARS-CoV-2 virus. Abandoning the pets roaming in the neighbourhood will increase the chances of the virus spreading. Thus, strong pets and animal management has been recommended. The infectious aerosol nature of SARS-CoV-2virus was confirmed when an infected bus passenger passed the virus to other fellow passengers in a bus [6] that had air circulating air conditioning working. The pattern of the COVID-19 virus outbreak on the Diamond Princess cruise ship established the role of poor ventilation in virus spread [7].

Authors in a review article [2] have discussed the viability and sanitization methods of disinfecting the virus in the air and on the surfaces. As a higher viral load was detected in the intensive care unit of the hospital treating COVID-19 patients, strong personal protective equipment is necessary for doctors and health care staff. In most populous country India, the dynamics of the virus spread till October 2020 has been described [5]. Here, the recent update of COVID-19 infections around the globe has been described briefly. In northern hemisphere, the second wave of coronavirus that began in fall and continued till winter was witnessed as the people stayed indoors and caused the risk of virus transmission. In November alone, nearly 105, 000 Europeans died of COVID-19 in 31 countries [8] in the second wave of infection. The number of people who died from novel coronavirus across Europe passed the 900, 000 mark on March 16, 2021 [9]. Across the US, the number of daily deaths reported increased substantially in November 2020. In the US, the daily death toll passed 3,000 which is the highest ever since the onset of the pandemic [10]. The number of cases in India after reaching a peak 97, 859 cases on September 16, 2020, showed a continuous decline. India registered [11] the lowest 8,635 cases in a day on February 2,2021 . The number of cases in India started [12] increasing again in the second wave, particularly in four states of Punjab, Maharashtra, Kerala, and Madhya Pradesh at the end of winter and start of spring. India recorded [13] the highest 35,886 fresh COVID-19 infections in 102 days on March 17 in the second wave of infection. The rise in coronavirus cases believed due to the discovery of several new variants of COVID-19 virus that is more contagious than the existing variants. But there is no scientific report to establish the new variant theory behind the surge, research is awaited. Recently, the state of Kerala on February 3 surpassed [14] Karnataka and became the second most affected state after Maharashtra in terms of the total number of cases. Currently, Kerala has $45 \%$ of India's total active cases. The current rise in coronavirus cases in Kerala is surprising as the state was successful in controlling the outbreak in the initial days of the pandemic. Also, because of its better health infrastructure, the viral caseload must have been the lowest among all the states, but it did not show such a pattern. However, the lowest case fatality rate (CFR) of Kerala has justified the better healthcare system of the state. The "Health Index Theory" put forward in this 
review article first established scientifically the reason for most coronavirus cases in Kerala compared to other states. The article is useful to contain the pandemic.

\section{RESULTS AND DISCUSSION}

\section{A. Caseload Analysis and Dynamics of Virus Spread}

During the initial stage, due to Kerala's strong COVID-19 specific measures (testing, tracking and tracing, quarantine, isolating, cluster monitoring, and stopping super spreading), the cases remained low. The state of Kerala was considered a 'success model' for the rest of the country. For example, on September 16, Kerala's caseload was just $3.4 \%$ compared to Maharashtra's $23.6 \%$ and the rest of India $73.0 \%$, as shown in Fig. 1 a. However, on February 12, 2021; the daily caseload of Kerala increased to $48 \%$, the highest in India, followed by Maharashtra $(23 \%)$ and the remaining $29 \%$ were from the rest of India as shown in the pie-chart (Fig. 1 b). In September, when cases were surging fast in the entire country, Kerala successfully contained the pandemic. But in the third week of November, it surpassed the caseload of Uttar Pradesh and Delhi. Later, in the first week of February 2021, it overtook Tamil Nadu, Andhra Pradesh, and Karnataka.

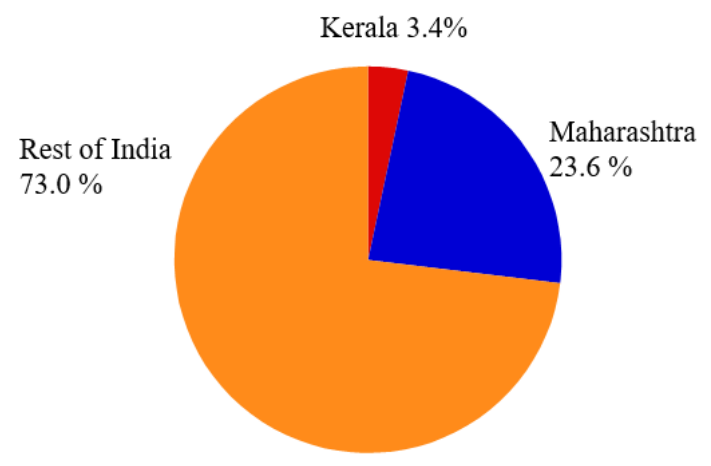

(a)

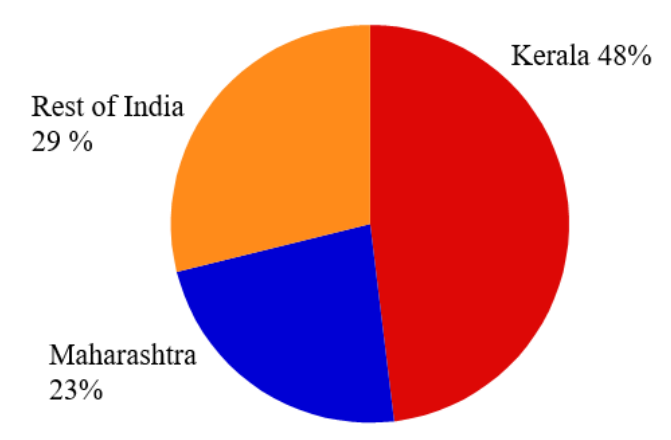

(b)

Fig. 1. Comparison of caseload of Kerala, Maharashtra, and rest of India on: (a) September 16, 2020;(b) February 12, 2021.

\section{B. Sero-survey Data}

The three sero-survey data of India and Kerala have been compared in Fig. 2. The first survey conducted in May 2020 showed that 0.33 and $0.73 \%$ population of Kerala and India, respectively developed antibodies. The results of the second (August) sero-survey were that $0.8 \%$ (Kerala) and $6.6 \%$ (India) population were infected. The data of the third
(December) survey shows that the antibodies were detected only in $11.6 \%$ of Kerala's population while the virus had infected $21.0 \%$ of Indian population. The sero-survey data show that the coronavirus did not spread in Kerala as widely as in the rest of India till January 2021 [14]. The transmission in Kerala became faster than the rest of India as the antibodies prevalence in Kerala was lesser (11.6\%) compared to India $(21.0 \%)$. On February 3, Kerala surpassed Karnataka and became the state with the highest number of infections among Karnataka, Andhra Pradesh, Tamil Nadu, Delhi, and Uttar Pradesh. Kerala is now second to Maharashtra in terms of the total number of cases. In terms of active cases, Kerala has the highest number among all the states of India. Kerala alone accounts for $45 \%$ of India's total active cases followed by Maharashtra (26\%) [14].

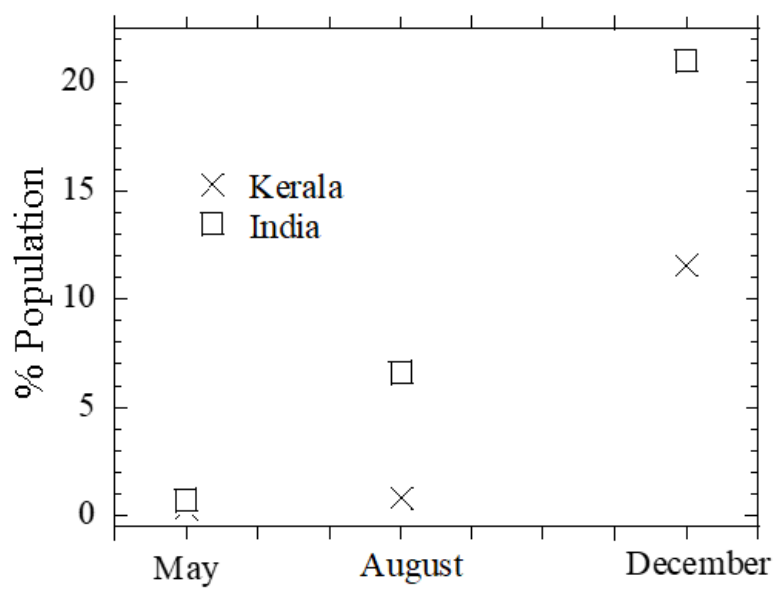

Fig. 2. Comparison of sero-survey data of Kerala and India.

\section{Health Index Theory}

III. The Health Index data of different states of India, prepared by the World Bank and Niti Aayog, have been shown in Fig. 3. Kerala is the best state in healthcare, while Uttar Pradesh ranked at the bottom [15]. Kerala's large infection rate can be explained by "Health Index Theory", the people living in higher health index state, better health infrastructure, have less immunity to pathogens including COVID-19 virus. On the other hand, people living in poor health infrastructure states are immune to several diseases and so to the SARS-CoV-2 virus. Because of the above health index theory, Kerala has the highest COVID-19 cases per million and Uttar Pradesh has the lowest (Figure 4). The hygiene hypothesis [16] stating that the people living in poor hygiene develop immunity for diseases and so for SARSCoV-2 virus, supports the "Health Index Theory" proposed in this article. However, the hygienic conditions and improved health infrastructure keep the population healthy and increase the life expectancy as can be seen in Figure 5. Kerala has the longest life expectancy while Uttar Pradesh has the shortest [17]. Due to better healthcare, the CFR of Kerala is the lowest (Figure 6) while Uttar Pradesh, with poor health infrastructure, has the second highest [18]. Figure 7 compares COVID-19 cases in Germany, Kerala, and France. The number of cases in Kerala was nearly the same as in Germany. However, the cases in France were 
more. The higher infection rate in Kerala comparable to the situation in Europe further supported the "Health Index Theory".

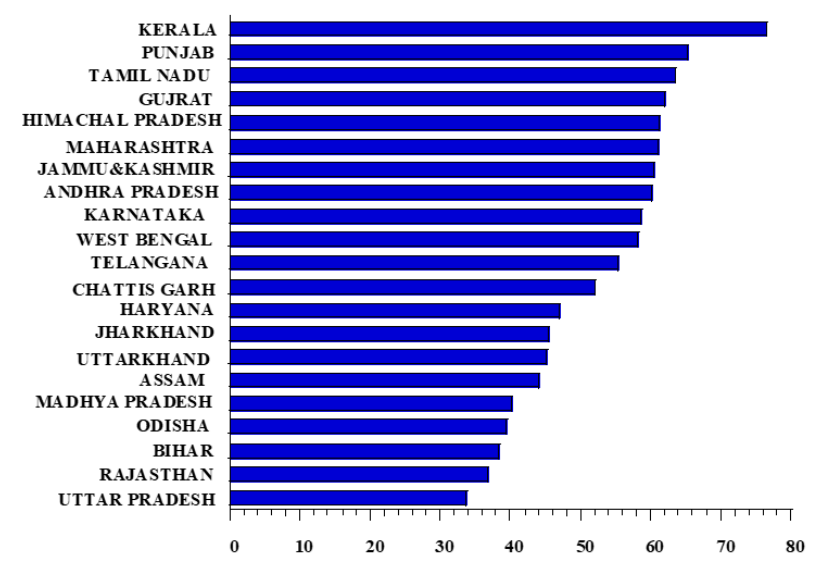

Fig. 3. Comparison of health index of different states of India.

\section{The Concept of Herd Immunity}

COVID-19 infections in Kerala are more (Fig. 4) compared to the other states of India and the same (Fig. 7) as in Germany. The dynamics of the virus spread in Kerala is also similar to Europe (unpublished data). As per the Health Index Theory, people in developed countries (Europe and North America) are more susceptible to the SARS-CoV2 virus. Therefore, cases in France and Germany are more than in India except in Kerala. The sero-survey results [19] of October 1, 2020, showed that $45 \%$ of the slums and $18 \%$ of the buildings' population in Mumbai have been exposed to the virus. The slum dwellers have reached herd immunity. So, recently in Mumbai, the virus is spreading faster in buildings and fewer or no infections in slums [20]. Similarly, in Kerala, fewer people have been infected than in other parts of the country, so virus transmission is more in Kerala than rest of the states. The relationship between new infection and sero-survey can be explained better by taking the example of coronavirus cases in Dharavi, the slums of Mumbai. Dharavi was once the major COVID-19 hotspot due to the dense population. On December 25, 2020, Dharavi reported zero cases [21]. Dharavi slums became COVID-19 free due to the herd immunity developed in the dwellers. The results of the sero-survey in Dharavi will likely show $70-80 \%$ antibodies prevalence. A survey conducted in October in the slums of Cuffe Parade, another slum also in Mumbai, showed that $75 \%$ population developed antibodies. Dharavi slums again on January 22, 2021, reported [22] no coronavirus cases for the second time, established the concept of herd immunity in the slums keeping the population safe from new infection. The recent surge in Kerala is due to because the virus did not penetrate as fast and deeper as it happened in other parts of the country which is happening now and thus resulting in a higher number of cases. This is supported by the sero-survey results as only $11.6 \%$ population was exposed to the virus in Kerala compared to $21.5 \%$ in the rest of the country. Also, the population of Kerala is more susceptible to the infection as compared to the other states of India, as explained by health index theory. Regarding the argument that the new coronavirus strains is responsible for the surge in the Kerala state, it has not been established yet. If the new strain is the cause, then a similar unexpected increase in other adjacent states could have been noticed. Research is needed to prove if the new more contagious mutation is the reason for the surge. The demographic reasons of high population density, elderly population, and prevalence of comorbidity and obesity may contribute to higher infection rate but only to a very small extent. There is very little evidence that 'letting their guard down' in Kerala is the reason for the increase in new cases.

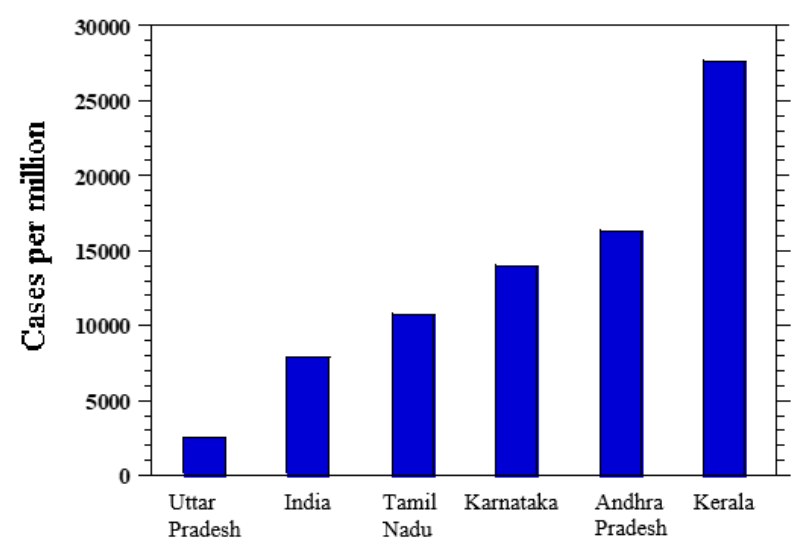

Fig. 4. COVID-19 cases per million (as on February 11, 2021).

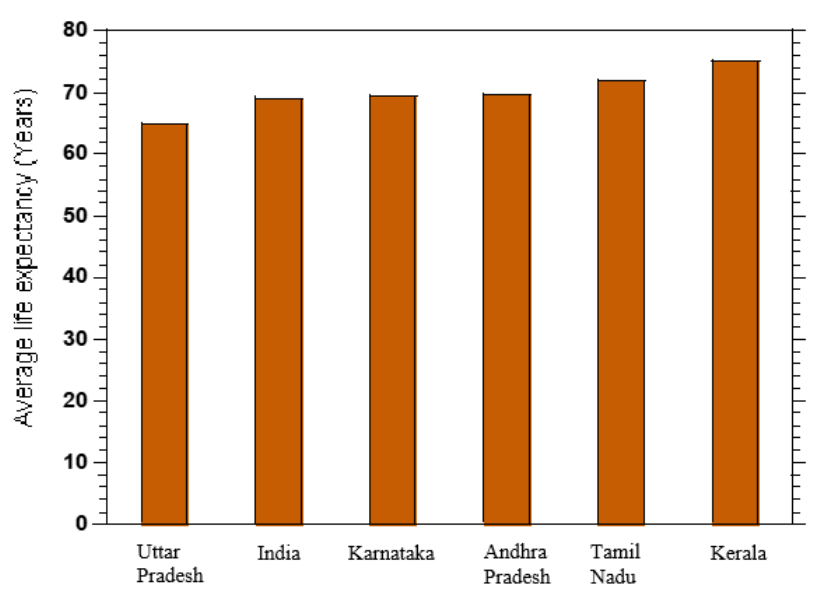

Fig. 5. Comparison of life expectancy of different states.

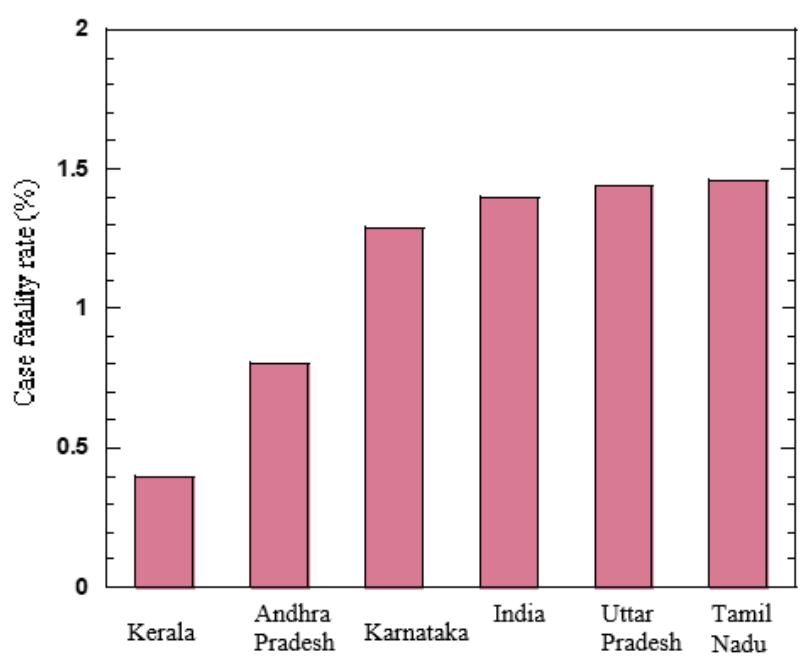

Fig. 6. Comparison of COVID-19 case fatality rate of different states (as on March 9, 2021). 


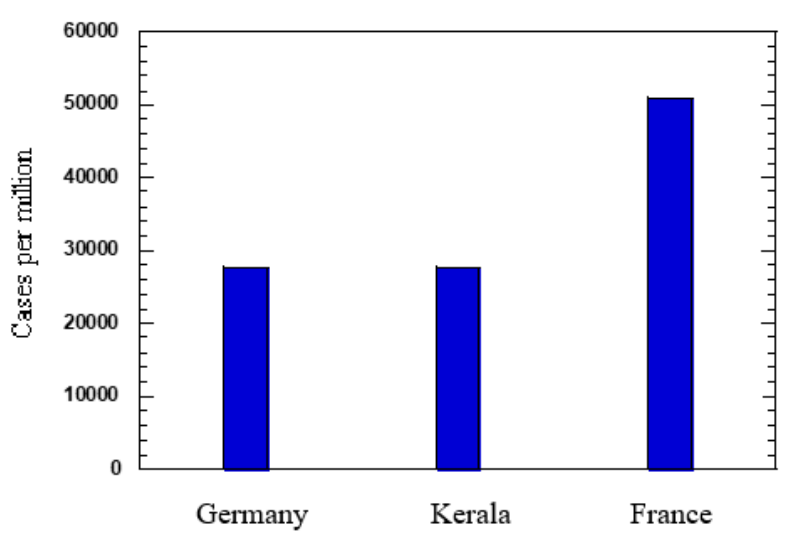

Fig. 7. Covid-19 cases per million (as on February 11, 2021).

\section{REFERENCES}

[1] Shervani Z, et al. SARS-CoV-2 Delayed Tokyo 2020 Olympics: Very Recent Advances in COVID-19 Detection, Treatment, and Vaccine Development Useful Conducting the Games in 2021. Advances in Infectious Diseases. 2020; 10: 56-66.

[2] Shervani Z, et al. Viability of SARS-CoV-2 and Sanitation Methods. European Journal of Medical and Health Sciences, 3(2021)22-27.

[3] Shervani Z, et al. COVID-19 Vaccine. Advances in Infectious Diseases. 2020;10: 195-210.

[4] Shervani Z, et al. World's Fastest Supercomputer Picks COVID-19 Drug. Advances in Infectious Diseases. 2020; 10: 211-225.

[5] Shervani Z, et al. Risk of SARS-CoV-2 Transmission from Humans to Pets and Vice Versa, European Journal of Medical and Health Sciences, 3(2021)34-38.

[6] Shen Y, et al. Community Outbreak Investigation of SARS-CoV-2 Transmission Among Bus Riders in Eastern China. Journal of the American Medical Association Internal Medicine. Published online,
September 1, 2020.

[7] Sakurai A, et al., Natural History of Asymptomatic SARS-CoV-2 Infection, The New England Journal of Medicine, Published on June $12,2020$.

[8] Josh Holder, Matina Stevis-Gridneff, and Allison McCann, Europe`s Deadly Second Wave: How Did It Happen Again? The New York Time, December 4, 2020.

[9] Covid-19 deaths in Europe pass 900,000: Report, The Times of India, March 16, 2021.

[10] Mike Hills, Covid-19 in the US: Bleak winter ahead as the deaths surge, BBC NEWS, 12 December, 2020

[11] Coronavirus live updates India reports 8,635 new cases, lowest in eight months, The Hindu, February 2, 2021.

[12] Shuja Asrar, India on brink of second Covid wave? At least 4 states of India witness fresh surge, TIMESOFINDIA.COM, February 20, 2021.

[13] India records 35,886 Covid-19 infections, highest in 102 days, The Times of India, March 18, 2021.

[14] Shuja Asrar, Covid cases: Why Kerala may be a 'victim` of its own `success`, TIMESOFINDIA.COM, February 12, 2021.

[15] Kerala best in healthcare, Tamil Nadu third: Health Index report, The New Indian Express, February 10, 2018.

[16] Rhythma Kaul, Poor hygiene, unsanitary conditions could lead to fewer deaths due to Covid-19: Study, Hindustan Times, October 28, 2020.

[17] Press Information Bureau, Government of India, Average Life Expectancy, 13 March, 2020

[18] Coronavirus (COVID-19) - Google News (JHU CSSE COVID-19 DATA).

[19] Krishna Kumar, Latest sero survey results come in as a dampener for BMC and Maharashtra government, The Economics Times, October $2,2020$.

[20] Pratip Acharya, Covid surge in Mumbai: More cases reported from buildings than slums, than in slums, The Free Press Journal, February 27, 2021.

[21] Chaitanya Marpakwar, Zero cases in Dharavi, first time since April 1, The Times of India, December 26, 2020.

[22] Chaitanya Marpakwar, Mumbai: Zero new Covid-19 cases in Dharavi second time, The Times of India, January 22, 2021. 